\title{
Index rerum ad Vol. 16
}

\section{Confecit Wilhelm Baumànn, Zurich}

Adenoma renalis, 77, 228 Adrenal glands, hormonal tumours, 193 Adrenogenitales Syndrom,

193 Aldosteronismus, 193

Bassinet, tonométrie, 1, 16

Bladder, cytologic study in cancer, 139

-, douple-contrast method, 292

-, dysfunction, 30

-, effect of Lisidonil, 46

- , instillations, 175

-, necrosis, 241

Calcules prostatiques, 148

Carcinoma prostatae, palpatio, 209

Carcinoma vesicae, cytologia, 139

Cushing Syndrom, 193

Cystitis interstitialis, instillationes, 175

Cystographia, 292

Cystometric studies of Lisidonil, 46

Cystoplasty, 280

Cytologic study in vesical cancer, 139

Dilatation prostatique, un nouvel instrument, 171

Doppelkontrastdarstellung der Harnbla-se, 292

Electroforesis sérida, tuberculosis uro-genital, 117

Ersatzblase aus dem Rectum, 280

Feocromocitoma, 193

Glucocorticoides en la tuberculosis de las vias urinarias, 158

Harnblase, Cytologie beim Carcinom,

139 -, Instillationen, 175 -, Nekrosen, $241 \sim$, Tumordarstellung durch Doppelkon-

trast, 292 Harnleiter, Druckmessung, 1, 16

-, Invaginationen, 268

-, traumatische Pseudocyste, 91 -

Harnleiterverschluß, Rolle des lympha-

tischen Systems, 341 Harnsediment, cytologische Befunde,

353 Homotransplantes intra-abdominales,

241 Horseshoe-kidney, conservative surgery,

129 Hydronephrosis experimentalis, 376

Intussuscepción ureteral, 268

Kidney, adenomatous new growths, 77 ,

228 -, measurement of pressure, 1, 16 -, modern surgery, 298 -, motor functions after resection, 319 -, necrosis, 241 
-, partial, transverse nephrectomy, 65 -, surgery in Horseshoe-kidney, 129 -, traumatic pseudocyst, 91

Lisidonil, 46 Liver, necrosis, 241

Megacystis, 30

Microscopie radiographique des concretions prostatiques, 148

Nebennieren, hormonbildende Tumoren 193

Necrosis in the kidney, liver and bladder, 241

Neo-vejiga rectal, 280

Nephrectomia partialis transversalis, 65

Neurologia urològica, 30

Niere, Chirurgie der Hufeisenniere, 129

-, adenomatöse Neubildungen, 228

-, Cysten nach Trauma, 91

-, Druckmessung im Nierenbecken, 1, 16

-, Hydrodynamik nach Teilresektionen, 319

394

Index rerum ad Vol. 16

XVI/1963

-, lymphatisches System, 341

-, moderne Chirurgie, 298

-, Nekrosen, 241

-, partielle Nephrektomie, 65

-, Prognose und Therapie von Adeno-

men, 77 Nierenbecken, Druckmessung, 1, 16

Osmic Tetroxide-fixed urinary sediment, 353

Pelvis renal, uréterotonografia, 1,16

Phaeochromocytom, 193

Predinisone, treatment of renal tuberculosis, 158

Presiòn ureteral, 1, 16

Prostata, calculi, 148

-, carcinoma, 209

Prostatatumoren, biochemische Teste, 180

Prostate, a new instrument for dilatation, 171

-, diagnosis of tumours, 180

Rein, chirurgie moderne, 298

-, necroses, 241

-, néo-formations adénomateuses, 228

-, néphrectomie partielle transversale,

65 -, pseudocyste traumatique, 91 -, resection partiale, 319 -, tonométrie du bassinet, 1, 16 -, traitement de l'adénome, 77 Rein en fer de cheval, chirurgie conser-

vatrice, 129 Renal pelvis, measurement of pressure,

1,16 Renal tuberculosis, treatment with pred-

nisone, 158 Retrocaval ureter in childhood, 103 Riñón, cirurgia moderna, 298 -, la nefrectomia parcial transversal, 65 -, necrosis, 241 
-, neoformaciones adenomatosas, 228 -, pseudoquiste traumatico, 91 -, resecciones parciales, 319 -, terapeutica del adenoma, 77 - en herradura, cirurgia conservadora,

129 Röntgenmikroskopie von Prostataver-

kalkungen, 148

Samenblase, Wirkung von Prolactin und Androsteron, 387

Sediment urinaire, examens cytologi-

ques, 353 Seminal vesicle, effect of Prolactin and

Androsterone, 387

Tertaplegia, neurologia urologica, 30 Tonométrie urétérale, 1, 16 Tuberculose urogénitale, éléctrophorèse

sérique, 117 -, glucocorticoides, 158

Ureter, pressure measurement, 1,16

-, traumatic pseudocyst, 91

-, bifidus, 365

-, retrocavo en la infancia, 103

Ureteral intussusception, 268

Ureteral obstruction, the role of the

lymphatic system, 341 Ureteral pressure, measurement, 1, 16 Uretère, pseudocyste, traumatique, 91 -, role du système lymphatique dans

Tobstruction, 341 Uretero-ileoplasty in Horseshoe-kidney,

129 Ureterotonographia, 1, 16 Urinary sediment, cytologic findings,

353 Urogenital tuberculosis, glucocorticoïde,

158 -, serum paper electrophoresis, 117 Urologia britànica, 257 Urological surgery, modern, 298

Urologie neurologique, 30

Vejiga, efecto del Lisidonil, 46

-, exámenes citológicos en el cancer, 139

-, instillación, 175

-, método de doble contraste, 292

-, necrosis, 241

-, neurologia urologica, 30

Vésicule séminale, $\Gamma$ effet de Prolactine

et de ГAndrostérone, 387 Vessie, Faction du Lisidonil, 46 -, dysfonctions, 30 -, examens

cytologiques dans le cancer,

1. $139-$, instillations

175 -, la méthode à double contraste

$292-$, necroses

241

X-Ray microscopy of prostatic concretions

148 